\title{
X-RAY EMISSION FROM DISTANT STELLAR CLUSTERS
}

\author{
Mean Luminosity versus Age in Late Type PMS Stars
}

\author{
N.S.SCHULZ, J.H.KASTNER \\ Massachusetts Institute of Technology \\ Cambridge MA 02143, USA
}

\section{Introduction}

Observations with the Einstein Observatory indicated that stellar X-ray activity diminishes in clusters older than $70 \mathrm{Myr}$ (Pleiades). ROSAT observations of older clusters also support this result (see Caillault 1995 and references therein). The timescales over which young stars diminish in $\mathrm{X}$ ray luminosity depends on spectral type (Randich et al. 1996), leading to the conclusion that X-ray activity in late type PMS depends on age and stellar mass. F and G-stars approach the main sequence much faster and the diminishing rates of $\mathrm{X}$-ray activity from $\mathrm{F}$ to $\mathrm{M}$ stars start to differ considerably. Kastner et al. (1997) observed that the mean of the ratio $L_{x} / L_{b o l}$ for $\mathrm{K}$ and $\mathrm{M}$ dwarf stars increases monotonically for low-mass stars from the very early $\mathrm{T}$ Tauri stage through the age of the Pleiades cluster, reflecting the contraction and spin-up of such stars during pre-main sequence evolution. This ratio then decreases towards middle aged stars, as late-type main sequence stars spin down. Here we extend this result by including more distant clusters that are younger overall than those considered by Kastner et al. and also including earlier spectral types.

\section{Results}

For very young stars we should be safe including $\mathrm{F}$ and G-types, for older ones the error grows significantly. Since we do not have much knowledge on the spectral types in most of the detected X-rays sources, we assume a random distribution from $\mathrm{F}$ up to early $\mathrm{M}$-type stars for an estimate of bolometric luminosities. Detailed photometric identifications clearly are an outstanding issue for the future. In figure 1 the estimated values of $L_{x} / L_{b o l}$ for IC 1396 (Schulz et al. 1997), the Rosette Nebula, NGC 2362 (Berghöfer 1997, priv. comm.) and IC 348 (Preibisch et al. 1996) are added to the ones 


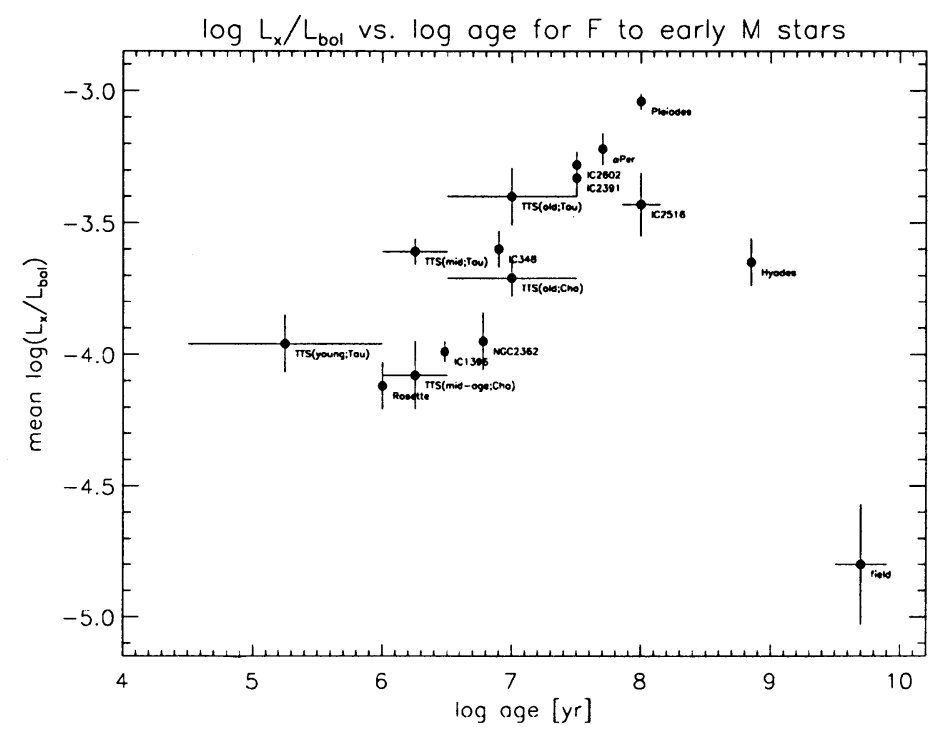

Figure 1. Mean of log of the ratio of X-ray to bolometric luminosity for late type PMS for F- up to early M-type stars. New entries are values for IC 1396, the Rosette Nebula, NGC 2362, IC 348 and IC 2516.

already shown in Kastner et al. (1997). For IC 348 figure 1 confines $L_{x} / L_{b o l}$ to an upper limit of -3.2 and thus allows us to set a mean $L_{x} / L_{b o l}$ of -3.6 . For completeness an estimate for an older cluster in IC 2516 (Dachs et al. 1996) was also added.

The mean values for very young clusters fit nicely into the trend observed $\mathrm{T}$ Tauri stars and nearby, somewhat older clusters. It is apparent that from the diagram one may be able to estimate the mean $L_{x} / L_{b o l}$ for clusters for which we are unable to determine the X-ray luminosity function directly. If we exercise this for IC 1805, which due to its distance is near the ROSAT sensitivity limit, we would get an expected mean value between -3.5 and -4.4 for an age of $1 \mathrm{Myr}$. The same would apply to $\operatorname{Tr} 14$, where no ROSAT pointing was found in the archive.

\section{References}

Caillault J.-P. (1996) , MPE report 263, ISSN 0178-0719, 67

Dachs J. , Hummel W. (1996), $A \& A$ 312, 818

Kastner J., Zuckerman B., Weintraub D.A., Forveille T., (1997), Science 277, 67

Preibisch T., Zinnecker H., Herbig G.H., (1996), A\&A 310, 456

Randich S., Schmitt J.H.M.M., Prosser C.F., Stauffer J.R., (1996), A\& A 300, 134

Schulz N.S., Berghöfer T., Zinnecker H., (1997), A\& A 325, 1001 